\title{
Resiliência e sua associação com religiosidade, espiritualidade e distúrbios afetivos em pacientes renais crônicos dialíticos
}

Resilience and its association with religiosity, spirituality and affective disorders in dialysis chronic kidney patients

\author{
La resiliencia y su asociación con la religiosidad, la espiritualidad y los trastornos afectivos en \\ pacientes renales crónicos en diálisis
}

Recebido: 14/05/2021 | Revisado: 23/05/2021 | Aceito: 07/06/2021 | Publicado: 21/06/2021

Claudia Maria Costa de Oliveira ORCID: https://orcid.org/0000-0002-2795-6681 Centro Universitário Christus, Brasil E-mail: claudiadrl@gmail.com

Amara Alçântara Gouveia

ORCID: https://orcid.org/0000-0003-3786-0648 Centro Universitário Christus, Brasil E-mail: amara.gouveia@ hotmail.com

Beatriz Carvalho Aragão Melo ORCID: https://orcid.org/0000-0003-1428-2603 Centro Universitário Christus, Brasil E-mail: beatrizaragaod@gmail.com

Maria Eduarda Coimbra Rocha Jucá ORCID: https://orcid.org/0000-0002-4542-040X Centro Universitário Christus, Brasil E-mail: eduardacoimbra7@gmail.com

Francisco Thiago Santos Salmito ORCID: https://orcid.org/0000-0001-6327-0022 Clínica Prontorim, Brasil E-mail: thiagosalmito@yahoo.com.br

Daniela Costa de Oliveira Santos ORCID: https://orcid.org/0000-0003-0472-4914

Centro Universitário Christus, Brasil E-mail: danisantos18@gmail.com Aline Moreira do Vale Mota ORCID: https://orcid.org/0000-0002-4345-2547

Centro Universitário Christus, Brasil E-mail: alinemvmota@gmail.com

Marcos Kubrusly

ORCID: https://orcid.org/0000-0002-4414-8109

Centro Universitário Christus, Brasil E-mail: mmkubrusly@gmail.com

\begin{abstract}
Resumo
Objetivos: avaliar o estado de resiliência e investigar sua associação com religiosidade/espiritualidade e distúrbios afetivos em diálise. Métodos: Estudo de centro único, transversal, com pacientes com idade $\geq 18$ anos, tempo de diálise $\geq 6$ meses. Os instrumentos da pesquisa foram a Escala de Resiliência-14 itens; Escala DUREL; Escala de Experiências Espirituais Diárias (EEED); e Escala de ansiedade, depressão e estresse (EADS). O estado de resiliência foi classificado e sua associação testada com a religiosidade, espiritualidade, estado de ansiedadeestresse-depressão dos pacientes. Resultados: Participaram 58 pacientes, 55,2\% masculino, idade média 51,5 anos. A resiliência foi muito baixa/baixa/no limite inferior em $15,5 \%$, moderada em $31 \%$, moderadamente alta/alta em $50 \%$ dos pacientes. Foi detectada diferença significativa nos escores de depressão, ansiedade, estresse e religiosidade intrínseca segundo o grau de resiliência, com pontuação mais elevada nos pacientes com resiliência mais baixa. A resiliência teve correlação linear negativa moderada com o escore de RI $(r=-0,407 ; p=0,002)$, de ansiedade $(r=-$ $0,360 ; p=0,006)$ e de depressão $(r=-0,316 ; p=0,016)$ e correlação negativa fraca com EEED $(r=-0,281 ; p=0,034)$. Não houve associação com idade, estado civil, nível econômico ou cultural, tipo de religião ou exames laboratoriais em diálise. Conclusão: A resiliência foi moderadamente alta/alta em 50\% dos pacientes em diálise, e quanto maior o nível de resiliência, observou-se estado afetivo mais positivo, melhor vivência plena da religiosidade e maior frequência nas experiências espirituais com Deus. Intervenções que possam auxiliar no manejo das dificuldades e adaptações à doença renal crônica devem ser promovidas.
\end{abstract}


Palavras-chave: Diálise renal; Resiliência psicológica; Espiritualidade; Religião; Ansiedade.

\begin{abstract}
Objectives: to evaluate the state of resilience and investigate its association with religiosity/spirituality and affective disorders in dialysis. Methods: Single-center, cross-sectional study with patients aged $\geq 18$ years, dialysis time $\geq 6$ months. The research instruments were the Resilience Scale-14 items; DUREL Scale; Daily Spiritual Experience Scale (DSES); and Anxiety, depression and stress scale (EADS). The state of resilience was assessed and its association tested with the patients'religiosity, spirituality, anxiety-stress-depression state. Results: 58 patients were included, $55.2 \%$ male, mean age 51.5 years. Resilience was very low /low/lower limit in $15.5 \%$, moderate in $31 \%$, moderately high/high in $50 \%$ of patients. A significant difference in the depression, anxiety, stress and intrinsic religiosity scores was detected according to resilience, with higher scores in patients with lower resilience. Resilience had a moderate negative correlation with the score of RI $(r=-0.407 ; p=0.002)$, anxiety $(r=-0.360 ; p=$ $0.006)$ and depression $(r=-0.316 ; p=0.016)$ and weak negative correlation with DSES $(r=-0.281 ; p=0.034)$. There was no association with age, marital status, economic or cultural level, type of religion or laboratory tests on dialysis. Conclusion: Resilience was moderately high/high in 50\% of dialysis'patients, and the higher the level of resilience, the more positive affective state, the better the full experience of religiosity and the greater frequency in spiritual experiences with God. Interventions that can assist in the management of difficulties and adaptations to chronic kidney disease should be promoted.
\end{abstract}

Keywords: Renal dialysis; Resilience, Psychological; Spirituality; Religion; Anxiety.

\begin{abstract}
Resumen
Objetivos: evaluar el estado de la resiliencia e investigar su asociación con la religiosidad / espiritualidad y los trastornos afectivos en diálisis. Métodos: Estudio unicéntrico, transversal, con pacientes $\geq 18$ años, tiempo de diálisis $\geq 6$ meses. Los instrumentos de investigación fueron la Escala de Resiliencia - 14 ítems; Escala DUREL; Escala de Experiencias Espirituales Diarias (EEED); y Escala de ansiedad, depresión y estrés (EADS). Se clasificó el estado de resiliencia y se probó su asociación con el estado de religiosidad, espiritualidad, ansiedad-estrés-depresión de los pacientes. Resultados: participaron 58 pacientes, $55,2 \%$ varones, edad media 51,5 años. La resiliencia fue muy baja / baja / en el límite inferior en el 15,5\%, moderada en el 31\%, moderadamente alta / alta en el 50\% de los pacientes. Se encontró una diferencia significativa en las puntuaciones de depresión, ansiedad, estrés y religiosidad intrínseca según el grado de resiliencia, con mayor puntuación en los pacientes con menor resiliencia. La resiliencia tuvo una correlación lineal negativa moderada con la puntuación de RI ( $r=-0,407 ; p=0,002)$, ansiedad $(r=-0,360 ; p=0,006)$ y depresión $(r=-0,316 ; p=0,016)$ y una correlación negativa débil con EEDS $(r=-0,281 ; p=0,034)$. No hubo asociación con la edad, estado civil, nivel económico o cultural, tipo de religión o pruebas de laboratorio en diálisis. Conclusión: La resiliencia fue moderadamente alta / alta en el 50\% de los pacientes en diálisis, y cuanto mayor era el nivel de resiliencia, se observaba un estado afectivo más positivo, mejor era la experiencia plena de religiosidad y mayor frecuencia en las experiencias espirituales con Dios. Se deben promover intervenciones que puedan ayudar en el manejo de las dificultades y adaptaciones a la enfermedad renal crónica.
\end{abstract}

Palabras clave: Diálisis renal; Resiliencia psicológica; Espiritualidad; Religión; Ansiedad.

\title{
1. Introdução
}

A hemodiálise (HD) é terapia renal substitutiva mais frequente no Brasil, abrangendo cerca de $90 \%$ dos pacientes com doença renal crônica em estágio terminal (DRET) (Marinho, 2017). A rotina de pacientes em HD inclui alterações físicas que resultam em limitações, exigindo uma adequação ao novo estilo de vida. O paciente com DRET se depara com situações cuja complexidade vai além do quadro clínico provocado pela doença, tendo que lidar com o sofrimento que ocorre da experiência de momentos difíceis como, rejeição, culpa e lutas, na tentativa diária de conviver em harmonia com sua condição de saúde. Essa convivência com a enfermidade e o tratamento dialítico acaba gerando conflitos que podem provocar angústia espiritual, agravando os sintomas físicos, emocionais e com isso a capacidade do enfrentamento da doença (Correia, et al., 2015).

Apesar das limitações e do sofrimento imposto pelo tratamento dialítico, alguns pacientes conseguem um melhor enfrentamento de sua doença, e vários fatores podem estar envolvidos (idade, sexo, estado socio-econômico-cultural, apoio familiar, condição psicológica), podendohaver também alguma contribuição da resiliência e da religiosidade do paciente. A resiliência é a característica que diferencia a forma como as pessoas lidam com os mesmos problemas, traduzindo a capacidade de um determinado indivíduo passar por uma situação adversa, conseguir superá-la e dela sair ileso (Fassbinder, 
et al., 2015). Pessoas resilientes apresentam autoestima positiva, habilidades de dar e receber em relações humanas, disciplina, responsabilidade, receptividade e tolerância ao sofrimento (Angst, 2009), o que pode colaborar para maior adesão ao tratamento hemodialítico e também melhor adaptação às restrições impostas pela doença.

A crença religiosa constitui uma parte importante da cultura, dos princípios e dos valores das pessoas, favorecendo a compreensão de eventos dolorosos, caóticos e imprevisíveis (Carone \& Barone, 2001). O estudo da religiosidade e da espiritualidade no contexto da saúde tem crescido de forma expressiva nas últimas décadas, tornando-se um ponto fundamental de estudos na área de saúde mental e coletiva (Martinez, et al., 2014). Dentre as várias formas de fortalecimento do paciente, evidencia-se a busca da espiritualidade e da religiosidade, recursos que ajudam as pessoas a lidar com situações traumáticas e estressantes relacionadas aos processos de enfermidade e internação (Ottaviani, et al., 2014). Praticar a religião é uma das características comumente encontradas na história de vida de pessoas resilientes (D’Souza \& Rodrigo, 2004).

Diante do exposto, os autores tiveram como objetivo do presente estudo avaliar o estado de resiliência de pacientes renais crônicos em hemodiálise, e investigar sua associação com características pessoais e do tratamento dialítico, com o estado de religiosidade e espiritualidade, bem como com estados afetivos negativos (estresse, ansiedade e depressão).

\section{Metodologia}

\section{Delineamento do estudo}

Trata-se de um estudo transversal, descritivo e quantitativo, desenvolvido em uma Unidade de Terapia Renal Substitutiva, que atende pacientes do Sistema Único de Saúde (SUS). Foram incluídos pacientes com mais de 18 anos, tempo superior a 6 meses de diálise, com capacidade de responder aos questionários da pesquisa e que assinaram termo de consentimento livre e esclarecido para participar do estudo. Foram excluídos pacientes com déficit cognitivo, visual ou outros. O estudo foi aprovado pelo Comitê de Ética em Pesquisa com o parecer número 3.319.283.

\section{Coleta de Dados}

Os dados clínicos foram coletados através de entrevista com os pacientes e revisão das pastas de acompanhamento da clínica de diálise. Os dados laboratoriais foram obtidos a partir do registro de computadores do centro. As seguintes variáveis socio-demográficas foram pesquisadas: idade; sexo; peso seco e altura, para cálculo do índice de massa corporal (IMC); doença renal primária; estado civil; nível cultural; rendimento familiar mensal em salários mínimos; atividade profissional. Em relação ao tratamento dialítico, foram pesquisados: tempo em hemodiálise; tipo de acesso vascular; encaminhamento para transplante renal; e variáveis laboratoriais (hemoglobina, ureia, creatinina, cálcio, fósforo, PTH e albumina).

A resiliência foi avaliada pela Escala de Resiliência de Wagnild e Young (1993), adaptada e validada para a língua portuguesa na sua versão original de 25 itens por Pesce et al. (2005) e que depois teve as propriedades psicométricas da versão brasileira de 14 itens testada por Damásio, Borsa e Silva (2011). Nesta versão, os itens são descritos de forma positiva, respondidos em uma escala tipo Likert variando de 1 (discordo totalmente) a 7 (concordo totalmente). As pontuações possíveis variam de 7 a 98, com pontos mais altos refletindo maior resiliência. A consistência interna da escala de resiliência de 14 itens tem sido reportada como excelente (alfa Cronbach $=0,93$ ) e correlaciona-se fortemente com a escala original de 25 itens $(r=0,97)$ (Wagnild, 2009). A resiliência na escala de 14 itens foi classificada como: 14-56= muito baixa, 57-64 = baixa, 65-73 = no limite inferior, 74-81= Moderada, 82-90= moderadamente alta e 91-98= alta (Wagnild, 2009a).

Para a avaliação da religiosidade, foi utilizada a versão traduzida e validada para o idioma português do Índice de Religiosidade de Duke (DUREL) (Taunay, et al., 2012), com alfa de Cronbach > 0,80. Este instrumento contém cinco itens que mensuram três dimensões do envolvimento religioso relacionadas a desfechos em saúde: Religiosidade Organizacional (RO) que refere-se à frequência aos encontros religiosos (exemplo: cultos, missas, cerimônias, grupos de oração ou de estudo, 
etc.); Religiosidade Não Organizacional (RNO) que trata da frequência de atividades religiosas privadas (exemplo: meditação, orações, leitura de textos religiosos, assistir ou ouvir programas religiosos na TV ou rádio, etc); e Religiosidade Intrínseca (RI) que se refere à busca de internalização e vivência plena da religiosidade como principal objetivo do indivíduo. As dimensões (RO, RNO e RI) são analisadas separadamente. Os domínios RO e RNO possuem pontuação de 1 a 6; o domínio RI tem pontuação de 3 a 15, considerando que menores pontuações correspondem a melhor índice de religiosidade.

Em relação à espiritualidade, foi utilizada a Escala de Experiências Espirituais Diárias (EEED), desenvolvida por Underwood e Teresi (2002) e, posteriormente traduzida e adaptada para a cultura brasileira (Kimura, et al., 2012), com uma boa consistência interna (alfa de Cronbach $=0,91$ ). É um instrumento que busca acessar a dimensão espiritual por meio das experiências que se manifestam na vida diária, como gratidão, compaixão, paz interior, conexão com o transcendente, sem envolvimento com crenças ou comportamentos de uma religião específica. Esta escala é composta por 16 itens, sendo que os 15 primeiros itens estão relacionados às experiências espirituais e distribuídos em uma escala do tipo Likert, com pontuação de que vai de um (1) para "muitas vezes ao dia" a seis (6) para quase nunca ou nunca. O item 16 refere-se à "proximidade com Deus" e possui somente quatro categorias de resposta, sendo que 1 refere-se a "nada próximo" e 4 indica "tão próximo quanto possível'. A pontuação total é obtida pela soma dos escores dos 16 itens, variando de 16 a 94 pontos. Vale ressaltar que para o cálculo da pontuação total, considera-se o item 16 com escore invertido, na qual a opção de categoria 1 será interpretada como (tão próximo quanto possível) e 4 (nada próximo). Na soma total, menores pontuações, refletem maior frequência as experiências espirituais e maior proximidade com Deus.

Para a avaliação da ansiedade, depressão e estresse foi utilizada a escala de depressão, ansiedade e estresse (EADS), adaptada e validada para a língua portuguesa, na versão curta de 21 itens por Apóstolo, Mendes e Azeredo (2006), a partir da versão original "The Depression Anxiety Stress Scales 21 (DASS-21)", que por sua vez é uma forma curta da escala DASS-42 itens de Lovibond e Lovibond (1995). A escala apresentou alfa de Cronbach de 0,90 para a depressão, 0,86 para a ansiedade, 0,88 para o estresse, respectivamente e 0,95 para o total das três sub-escalas. Cada uma das dimensões da escala inclui sete itens, no total de 21 itens. Cada item consiste em uma afirmação que remete para sintomas emocionais negativos. Pede-se ao sujeito que responda se a afirmação se lhe aplicou "na semana passada". Para cada frase existem quatro possibilidades de resposta, apresentadas numa escala tipo Likert de 4 pontos: "não se aplicou nada a mim", "aplicou-se a mim algumas vezes", “aplicou-se a mim muitas vezes", “aplicou-se a mim a maior parte das vezes". A escala fornece três notas, uma por subescala, em que o mínimo é " 0 " e o máximo " 21 ". As notas mais elevadas em cada escala correspondem a estados afetivos mais negativos.

Os instrumentos da pesquisa foram aplicados previamente à sessão de hemodiálise ou nas duas primeiras horas de tratamento, sob a forma de entrevista individual, no período de julho a novembro de 2019.

\section{Análise estatística}

Os resultados das variáveis contínuas foram expressos em média \pm desvio padrão e das variáveis categóricas em percentuais. As variáveis contínuas com distribuição normal foram comparadas através do teste T de Student ou ANOVA e aquelas com distribuição anormal pelo teste de Mann-Whitney. As variáveis categóricas foram comparadas pelo qui-quadrado ou teste exato de Fisher. Para testar a associação entre resiliência e as variáveis do estudo, os pacientes foram divididos em dois grupos, tendo como ponte de corte o percentil 25 da escala de resiliência. Um valor de p inferior a 5\% foi considerado estatisticamente significante. Foi utilizado o coeficiente de correlação de Pearson para verificar a existência e a magnitude da correlação entre o escore de resiliência e as variáveis clinicas, os escores de RO, RNO, RI, EEED e os escores de depressão, estresse e ansiedade. A magnitude das correlações foi classificada como: fraca $(r<0,3)$; moderada $(0,3$ a 0,59$)$; forte $(0,6$ a 0,9) e ideal $(1,0)$ (Levin \& Fox, 2004). Os dados foram registrados em uma planilha de Microsoft Excel 10.0 e transferidos 
para o programa SPSS versão 21.0, onde a análise estatística foi realizada.

\section{Resultados}

Participaram do estudo 58 pacientes em hemodiálise, sendo 55,2\% do sexo masculino e com idade média de 51,5 anos. As características sociodemográficas da população em estudo encontra-se demonstrada na Tabela 1. A etiologia da DRET foi hipertensão arterial sistêmica em 31,0\% dos casos, Indeterminada (29,4\%), diabetes mellitus (25,8\%), nefrolitíase $(1,7 \%)$, nefrite lúpica $(1,7 \%)$ e outras $(10,3 \%)$. Os resultados de exames laboratoriais realizados de rotina pela clínica de diálise estão demonstrados na Tabela 2. 
Tabela 1. Distribuição dos pacientes em estudo, segundo as variáveis sociodemográficas.

\begin{tabular}{|c|c|c|}
\hline Variável clínica & Média \pm DP & Variação \\
\hline Idade (anos) & $51,5 \pm 13,0$ & $22-76$ \\
\hline Tempo HD (meses) & $63,5 \pm 58,1^{*}$ & $4,3-256,7$ \\
\hline Peso seco $(\mathrm{kg})$ & $66,7 \pm 18,5$ & $44-123$ \\
\hline Altura (metros) & $1,59 \pm 0,1$ & $1,36-1,82$ \\
\hline $\mathrm{IMC}(\mathrm{kg} / \mathrm{m} 2)$ & $26,1 \pm 6,4$ & $18-42,1$ \\
\hline Renda familiar (reais) & $1852 \pm 1251$ & $1000-8360$ \\
\hline Variável clínica & $\mathbf{N}$ & $\%$ \\
\hline \multicolumn{3}{|l|}{ Sexo } \\
\hline Feminino & 26 & $44,8 \%$ \\
\hline Masculino & 32 & $55,2 \%$ \\
\hline \multicolumn{3}{|l|}{ Estado Civil } \\
\hline Solteiro & 19 & $32,8 \%$ \\
\hline Casado & 31 & $53,4 \%$ \\
\hline Divorciado & 04 & $6,9 \%$ \\
\hline Viúvo & 04 & $6,9 \%$ \\
\hline \multicolumn{3}{|l|}{ Raça } \\
\hline Branca & 07 & $12,1 \%$ \\
\hline Parda & 30 & $51,7 \%$ \\
\hline Negra & 11 & $19,0 \%$ \\
\hline Não classificado & 10 & $17,2 \%$ \\
\hline \multicolumn{3}{|l|}{ Religião } \\
\hline Católica & 33 & $56,9 \%$ \\
\hline Evangélica & 21 & $36,2 \%$ \\
\hline Espírita & 01 & $1,7 \%$ \\
\hline Testemunha Jeová & 01 & $1,7 \%$ \\
\hline Ateu & 02 & $3,4 \%$ \\
\hline \multicolumn{3}{|l|}{ Nível cultural } \\
\hline Analfabeto & 29 & $50,0 \%$ \\
\hline Fundamental & 25 & $43,1 \%$ \\
\hline Superior & 04 & $6,9 \%$ \\
\hline \multicolumn{3}{|l|}{ Profissão } \\
\hline Aposentado & 29 & $50,0 \%$ \\
\hline Benefício & 20 & $34,5 \%$ \\
\hline Trabalho ativo & 03 & $5,2 \%$ \\
\hline Trabalho doméstico & 02 & $34,5 \%$ \\
\hline Desempregado & 04 & $6,9 \%$ \\
\hline \multicolumn{3}{|l|}{ Acesso atual } \\
\hline FAV & 42 & $72,4 \%$ \\
\hline Cateter temporário & 09 & $15,5 \%$ \\
\hline Cateter permanente & 07 & $12,1 \%$ \\
\hline \multicolumn{3}{|l|}{ Encaminhamento transplante } \\
\hline Sim & 50 & $87,7 \%$ \\
\hline Não & 07 & $12,3 \%$ \\
\hline
\end{tabular}

HD: hemodiálise; IMC: índice de massa corporal; FAV: fístula arteriovenosa *mediana do tempo em diálise: 48,2 meses (percentil 25: 19,8 e 75: 90,7 meses) Fonte: dados da pesquisa. 
Tabela 2. Exames laboratoriais realizados na rotina do tratamento dialítico da população em estudo.

\begin{tabular}{lll}
\hline Variável laboratorial & Média $\pm \mathrm{DP}$ & Variação \\
\hline Hemoglobina $(\mathrm{g} / \mathrm{dl})$ & $11,5 \pm 1,7$ & $7,4-15,2$ \\
Ureia $(\mathrm{mg} / \mathrm{dl})$ & $112,5 \pm 30,8$ & $65-187$ \\
Creatinina $(\mathrm{mg} / \mathrm{dl})$ & $8,8 \pm 2,9$ & $2,7-17,2$ \\
Cálcio $(\mathrm{mg} / \mathrm{dl})$ & $9,0 \pm 0,8$ & $6,9-11,9$ \\
Fósforo $(\mathrm{mg} / \mathrm{dl})$ & $5,5 \pm 1,7$ & $2,7-10,0$ \\
PTH $(\mathrm{pg} / \mathrm{ml})$ & $279,7 \pm 200,8^{* *}$ & $56,0-890,0$ \\
Albumina $(\mathrm{g} / \mathrm{dl})$ & $3,8 \pm 0,4$ & $2,8-4,7$ \\
\hline
\end{tabular}

DP: desvio padrão

**PTH: mediana $=226$ pg/ml (percentil 25: 120; percentil 75: 408).

Fonte: dados da pesquisa.

A classificação do estado de resiliência encontra-se no Gráfico 1. O escore médio da resiliência foi de $81,0( \pm 11,3)$ pontos, mediana de 81,5 (percentil 25: 76 e percentil 75: 90), com valor mínimo de 41 e máximo de 98 pontos. A resiliência na escala de 14 itens foi classificada como: muito baixa em 3,4\%, baixa em 6,9\%, no limite inferior em 8,6\%, moderada em $31 \%$, moderadamente alta em $27,6 \%$ e alta em $22,4 \%$ dos pacientes.

Gráfico 1. Classificação da resiliência na população em estudo, segundo a Escala de Resiliência de 14 itens.

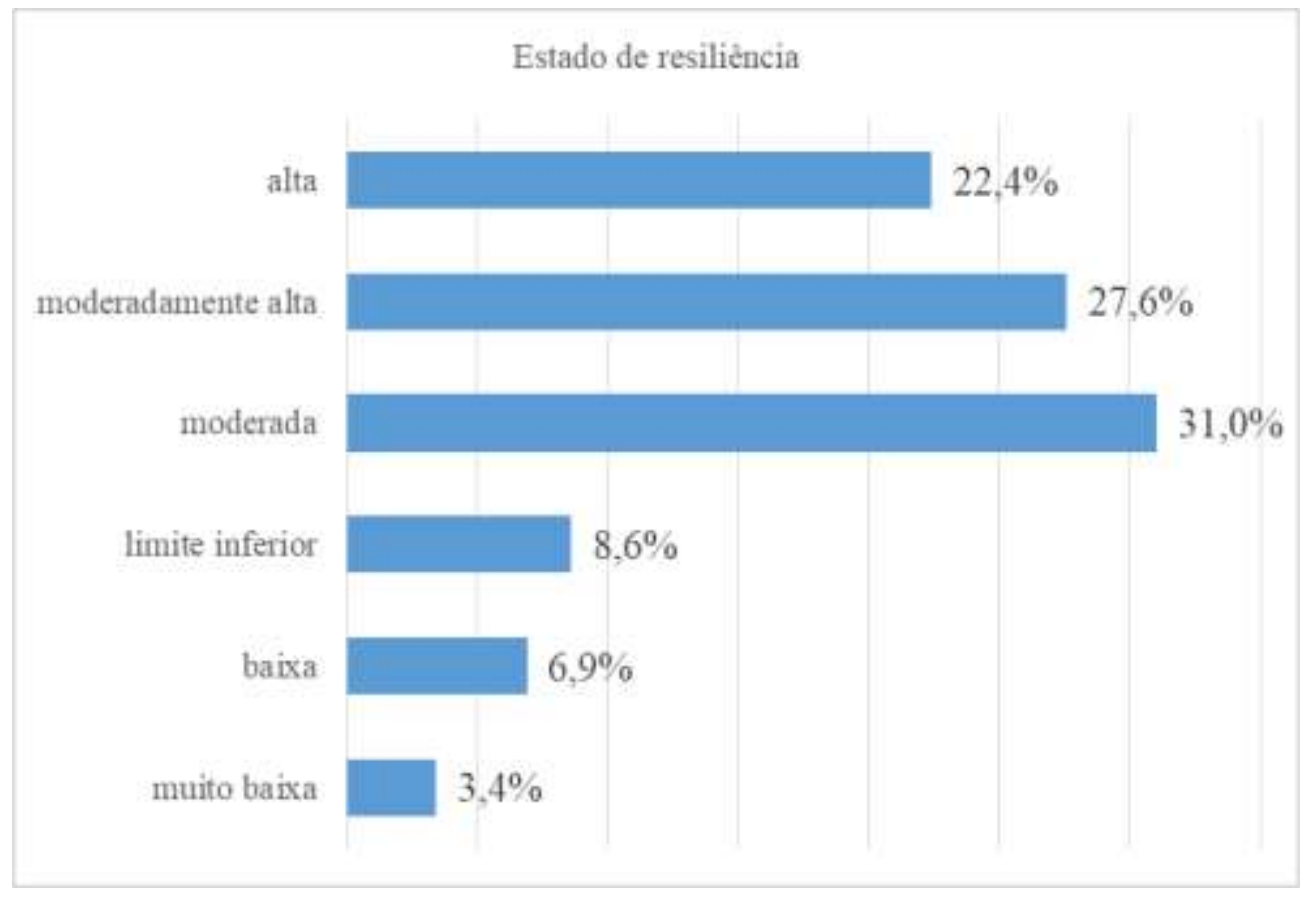

Fonte: Dados da pesquisa.

Os dados referentes aos escores da Escala de religiosidade de DUREL estão apresentados na Tabela 3. 
Tabela 3. Avaliação da religiosidade pela Escala de DUREL na população em estudo.

\begin{tabular}{llll}
\hline & Média \pm DP & Mediana & Variação do domínio \\
\hline Religiosidade Intrínseca & $4,97 \pm 2,21$ & 4,0 & 3 a 12 \\
Religiosidade Organizacional & $2,69 \pm 1,29$ & 2,0 & 1 a 6 \\
Religiosidade Não Organizacional & $2,43 \pm 1,29$ & 2,0 & 1 a 6 \\
\hline
\end{tabular}

DP: desvio padrão

Fonte: Dados da pesquisa

O escore total da EEED foi de 32,5 ( $(10,5)$ pontos, com variação de 18 a 70 . A mediana foi de 31 pontos (percentil $25=25,7$ e percentil $75=36,2$ ), refletindo moderada frequência de experiências espirituais diárias. Considerando os resultados da escala EADS-21, os resultados estãodemonstrados na Tabela 4.

Tabela 4. Avaliação da Escala de ansiedade, depressão e estresse (EADS-21) na população em estudo.

\begin{tabular}{lllll}
\hline ESCORE & Média \pm DP & Mediana & Variação & Percentil 75 \\
\hline Ansiedade & $1,74 \pm 2,03$ & 1,0 & $0,0-10,0$ & 2,25 \\
Depressão & $1,34 \pm 1,87$ & 1,0 & $0,0-8,0$ & 2,00 \\
Estresse & $1,77 \pm 2,36$ & 1,0 & $0,0-10,0$ & 3,00 \\
\hline
\end{tabular}

DP: desvio padrão

Fonte: Dados da pesquisa

Não houve diferença significativa na classificação da resiliência em dois grupos (ponto de corte: percentil 25) e a idade, sexo, estado civil, tipo de acesso vascular, renda familiar, nívelde instrução, exames laboratoriais, pontuação da EEED e domínios da RO e RNO, sendo observada diferença significativa para os escores de depressão, ansiedade, estresse e RI, com pontuação mais elevada e portanto mais negativa nos pacientes com resiliência mais baixa (Tabela 5).

Tabela 5. Associação entre o estado de resiliência e as variáveis do estudo.

\begin{tabular}{llll}
\hline Variável & $<\mathbf{7 6}(\mathbf{n}=\mathbf{1 3})$ & $\mathbf{7 6 - 9 8}(\mathbf{n = 4 5})$ & $\mathbf{p}$ \\
\hline Idade (anos) & $52,2 \pm 16,8$ & $51,3 \pm 12,1$ & 0,858 \\
Tempo em diálise & $46,4 \pm 35,6$ & $69,1 \pm 63,1$ & 0,107 \\
IMC (kg/m $)$ & $24,5 \pm 2,9$ & $26,5 \pm 7,1$ & 0,171 \\
Renda familiar (reais) & $1639,23 \pm 722,42$ & $1936,40 \pm 1378,71$ & 0,460 \\
Hb (g/dl) & $11,15 \pm 1,49$ & $11,11 \pm 3,02$ & 0,963 \\
Ureia (mg/dl) & $100,07 \pm 23,99$ & $115,26 \pm 35,58$ & 0,118 \\
Creatinina (mg/dl) & $8,76 \pm 2,96$ & $9,04 \pm 0,92$ & 0,964 \\
Albumina (g/dl) & $3,76 \pm 0,32$ & $3,77 \pm 0,36$ & 0,921 \\
Cálcio (mg/dl) & $8,85 \pm 0,42$ & $9,04 \pm 0,92$ & 0,316 \\
Fósforo (mg/dl) & $5,13 \pm 1,99$ & $5,57 \pm 1,52$ & 0,410
\end{tabular}




\begin{tabular}{|c|c|c|c|}
\hline PTH & $256,5 \pm 154,6$ & $261,36 \pm 218,3$ & 0,941 \\
\hline RO & $2,79 \pm 1,64$ & $2,66 \pm 1,47$ & 0,819 \\
\hline RNO & $2,92 \pm 1,65$ & $2,29 \pm 1,17$ & 0,221 \\
\hline RI & $6,15 \pm 2,37$ & $4,61 \pm 2,09$ & $\mathbf{0 , 0 2 8}$ \\
\hline EEED & $35,54 \pm 9,73$ & $31,52 \pm 10,7$ & 0,231 \\
\hline Escore depressão & $2,38 \pm 2,39$ & $1,09 \pm 1,63$ & $\mathbf{0 , 0 4 0}$ \\
\hline Escore estresse & $3,07 \pm 3,49$ & $1,29 \pm 1,67$ & 0,013 \\
\hline Escore ansiedade & $3,07 \pm 2,56$ & $1,34 \pm 1,72$ & 0,006 \\
\hline Sexo & & & 0,850 \\
\hline Feminino & $24,0 \%$ & $76,0 \%$ & \\
\hline Masculino & $21,9 \%$ & $78,1 \%$ & \\
\hline Acesso vascular & & & 0,593 \\
\hline FAV & $26,2 \%$ & $73,8 \%$ & \\
\hline Cateter temporário & $12,5 \%$ & $87,5 \%$ & \\
\hline Cateter permanente & $14,3 \%$ & $85,7 \%$ & \\
\hline Estado civil & & & 0,469 \\
\hline Solteiro & $33,3 \%$ & $66,7 \%$ & \\
\hline Casado & $19,4 \%$ & $80,6 \%$ & \\
\hline Divorciado/Viúvo & $12,5 \%$ & $87,5 \%$ & \\
\hline
\end{tabular}

IMC: índice de massa corpórea; Hb: hemoglobina; RO: religiosidade organizacional; RNO: religiosidade não organizacional; RI: religiosidade intrínseca; EEED: escala de experiências espirituais diárias, FAV: fístula arterio-venosa Tests estatisticos: teste t Student, Mann-Whitney, teste exato de Fisher e qui-quadrado Fonte: dados da pesquisa

A resiliência teve correlação negativa moderada com o escore de $\mathrm{RI}(\mathrm{r}=-0,407 ; \mathrm{p}=0,002)$, ansiedade $(\mathrm{r}=-0,360 ; \mathrm{p}=$ $0,006)$ e depressão $(r=-0,316 ; p=0,016)$ e correlação negativa fraca com o escore da $\operatorname{EEED~}(r=-0,281 ; p=0,034)$.

\section{Discussão}

A resiliência pode ter um papel no enfrentamento da hemodiálise (HD), que é um tratamento difícil, de longo prazo e que costuma causar tensão e ansiedade nos pacientes. No presente estudo, observou-se elevado percentual (81\%) de pacientes com resiliência alta/moderadamente alta/moderada. Segundo Santos e Costa (2016), estudando pacientes em HD em um centro de Itajubá, $61 \%$ apresentavam pontuação da resiliência acima da média, indicando probabilidade de um melhor enfrentamento da doença. Entretanto, estes autores utilizaram a escala de resiliência de 25 itens (pontuação de 25-175) e não descreveram o escore médio de resiliência no grupo de 61 participantes, dificultando a comparação com os resultados do presente estudo Neste mesmo estudo, a tendência à resiliência acima da média foi mais prevalente nos pacientes com prática religiosa. Gomes, Lanzoti e Orlandi (2017), em estudo com 100 pacientes em HD em São Paulo, observaram um escore médio de resiliência de 131,38 $( \pm 20,62)$ pontos, o que foi considerado bastante satisfatório. Uma correlação negativa moderada foi detectada entre o escore de resiliência e a idade ( $r=-0.314 ; \mathrm{p}<0,001)$ e o tempo de diálise $(r=-0,317 ; \mathrm{p}=0,001)$ e uma 
correlação positiva fraca com o nível educacional $(r=0,222 ; \mathrm{p}=0,027)$, diferente do presente estudo que não detectou associação entre resiliência e estas variáveis. Boell, Silva e Hegadoren (2016), avaliando 191 pacientes com DRC no sudeste do Brasil, observaram um escore médio de resiliência de 67,5 ( \pm 15,4) pontos, tendo sido utilizada a Escala de Resiliência desenvolvida por Connor e Davidson (CD-RISC) (2003), cujos escores oscilam de zero a 100 pontos. As variáveis que influenciaram a resiliência neste estudo foram a duração da doença, a religião e o IMC.

Duran, Avci e Esim (2020), utilizando um instrumento diferente para avaliar a resiliência de 134 pacientes de dois centros de diálise da Turquia, com escores variando de 33-165 pontos, evidenciaram que o escore de resiliência médio foi de 100,65 $( \pm 18,54)$ pontos, considerado um nível moderado de resiliência. O escore foi significativamente inferior nos pacientes em diálise com idade entre 22-44 anos, solteiros, somente alfabetizados, com baixos salários e com duração da diálise superior a 5 anos. Os níveis de resiliência foram positivamente correlacionados com os níveis educacional, econômico e de espiritualidade, e negativamente correlacionados com a duração da diálise. Um estudo realizado em 3 centros de diálise de Taiwan, com 195 pacientes idosos acima de 60 anos, detectou um escore médio de resiliência de 116,72 $( \pm 31,52)$, sendo 46,4\% classificados como resiliência baixa e 36,6\% moderada. Esse estudo utilizou a versão chinesa da Escala de Resiliência de Wagnild de 25 itens, tendo sido a resiliência classificada como alta, moderada ou baixa quando escores entre 147-175, 127-146 e < 120, respectivamente (Liu, et al., 2018). A baixa resiliência associou-se como maiores escores de depressão e com comportamentos associados a promoção de menores níveis de saúde.

Em relação às características religiosas, identificamos que a maioria dos pacientes dialíticos do presente estudo era católica (56,9\%), dado que se assemelha ao estudo de Souza, Devezas e Santos (2018), no qual o catolicismo foi a filiação religiosa mais frequente, abrangendo 57,1\% da amostra. De acordo com o Censo do IBGE de 2010, 64,6\% da população geral do Brasil era católica, embora no final do ano de 2019, este percentual tenha caído para 50\%.

Quanto à religiosidade, no presente estudo os valores médios referentes à RO e RNO foram de 2,69 e 2,43 respectivamente (em uma escala que pode variar de 1 a 6), e de 4,9 para a RI (em uma escala que pode variar de 3 a 15). Souza, Devezas e Santos (2018), utilizando a mesma escala para avaliação da religiosidade de 21 pacientes em HD, encontraram valores médios de 3,06, 2,76 e 5,41 para RO, RNO e RI, respectivamente. No presente estudo, foi observada uma correlação negativa moderada entre resiliência e RI $(r=-0,407 ; p=0,002)$. Menores pontuações correspondem a melhor índice de religiosidade e portanto, quanto mais resiliente o paciente, menor o escore de religiosidade e portanto mais religiosidade ele apresenta. A religiosidade intrínseca está ligada a um sentimento de significado na vida, enquanto a religiosidade extrínseca relaciona-se a comportamentos religiosos visando benefícios pessoais, segurança ou distração. Berman et al. (2004), estudando 74 pacientes em HD nos Estados Unidos, e usando a escala "Hoge Intrinsic Religiosity Scale" encontraram que pacientes com alta religiosidade intrínseca (característica do indivíduo realmente religioso, que tem suas crenças internalizadas na vida diária) possuíam alta satisfação nas suas vidas e aqueles que possuíam altos valores na escala de religiosidade organizacional (frequência religiosa) possuíam mais satisfação para com os cuidados médicos. Diferentemente, a correlação entre resiliência e RI foi positiva e fraca $(r=0,218 ; p=0,002)$ e com a RO foi negativa e fraca ( $r=-0,253 ; p<0,001)$, no estudo de Freire de Medeiros et al. (2016), que incluiu 205 pacientes de um centro de diálise de Fortaleza e utilizou a mesma Escala de Durel. Entretanto, neste estudo os autores inverteram a pontuação do Likert no sentido positivo, justificando a correlação positiva com a RI.

Em revisão de literatura de artigos que discutiam a relação entre espiritualidade, religiosidade e saúde em pacientes dialíticos, maiores níveis de espiritualidade e religiosidade associaram-se com melhor qualidade de vida, menor prevalência de depressão, maior suporte social, mais satisfação com a vida e mais satisfação com o tratamento médico provido pelo nefrologista (Lucchetti, Almeida, Granero, 2010). Santos et al. (2017) acreditam que o uso de recursos religiosos e intervenção psicoespiritual devem ser encorajados em diálise, uma vez que o enfrentamento positivo para religiosidade e 
espiritualidade estiveram associados com maior qualidade de vida e menor depressão entre pacientes em HD. Os autores encontraram um prevalência de depressão de 27,3\% entre 161 pacientes de dois centros de diálise, utilizando a versão de 20 itens da "Center for Epidemiological Scale-Depression” (CES-D).

Atualmente não há consenso em como definir religiosidade e espiritualidade, bem como na forma incluir recomendações específicas nas diretrizes em nefrologia. Burlaco et al. (2019), em uma revisão sistemática sobre religiosidade (R), espiritualidade (E) e qualidade de vida, identificaram 50 artigos com um total de 9265 pacientes para inclusão na revisão. De uma lista de 177 escalas de religiosidade/espiritualidade disponíveis mundialmente, os autores identificaram 24 instrumentos, os demais sendo entrevistas, escalas ou questionários não padronizados. Em todos os estudos, religiosidade e espiritualidade estiveram positivamente correlacionadas com pelo menos uma variável da qualidade de vida. Os autores sugerem que a falta de consenso acima descrita pode dever-se ao fato de que R/E são fenômenos difíceis de medir e avaliar do ponto de vista sociológico e psicológico.

A espiritualidade é responsável por promover o sentimento de esperança, além de funcionar como ferramenta de apoio e fortalecimento, o que é muito importante para os indivíduos que fazem HD (Ottaviani, et al., 2014). Existem várias escalas capazes de avaliar a espiritualidade, tendo sido escolhida a EEED no presente estudo. Forti, Serbena e Scaduto (2020) em revisão sistemática recente sobre saúde e espiritualidade/religiosidade, com foco em instrumentos para sua mensuração, identificaram 35 trabalhos, descrevendo o uso de 16 instrumentos de mensuração de espiritualidade/religiosidade, entre eles o Índice de Religiosidade de Duke e a Escala de experiências espirituais diárias (EEED), aqui utilizadas. Souza, Devezas e Santos (2018) também utilizaram a escala EEED, obtendo um escore médio de 43,5, enquanto no nosso estudo encontramos uma média de 32,5, o que portanto representa uma frequência maior de experiências espirituais. Segundo Siqueira, Fernandes e Moreira-Almeida (2018), a religiosidade, a espiritualidade e o senso de coerência estão relacionados à felicidade e a uma maior qualidade de vida em pacientes com DRC em HD, aspectos não avaliados no presente estudo.

De acordo com Bujang et al. (2015), a prevalência de sintomas psicológicos entre os pacientes em HD é significativamente elevada,especialmente a depressão e a ansiedade. Uma revisão sistemática de 59 estudos de pacientes em diálise identificou uma prevalência de depressão de $27 \%$ (variando de 7-58\%) e ansiedade de $38 \%$ (variando de 12-52\%) (Murtagh, Addington-Hall, Higginson, 2007), sendo os nossos resultados ligeiramente superiores (depressão 36,3\% e ansiedade 46,6\%). Oliveira et al. (2012), avaliando 104 pacientes em diálise em Fortaleza, evidenciaram depressão em 65,3\% dos pacientes (utilizando o inventário de depressão de Beck), tendo sido a depressão associada com menor qualidade de vida. Altos índices de depressão em pacientes renais crônicos também merecem atenção no que se refere à capacidade de resiliência desses pacientes (Zimmermann, Carvalho, Mari, 2004). Uma revisão sistemática sobre resiliência e doença crônica evidenciou uma correlação inversa entre resiliência e depressão, ansiedade, incapacitação, somatização e progressão da doença, ao mesmo tempo que detectou uma associação positiva entre resiliência e qualidade de vida e comportamento que leva à promoção de saúde (Cal, et al., 2015).

O presente estudo não evidenciou associação do estado de resiliência de pacientes em diálise com dados demográficos, tipo de acesso vascular, nível de instrução, exames laboratoriais, pontuação da EEED e domínios da religiosidade, mas foi observada diferença nos escores de depressão entre os grupos de resiliência, commaior pontuação nos pacientes com resiliência baixa, ou seja, quanto menor a resiliência maior o escore de depressão, o que sinaliza que um estado afetivo negativo pode estar associado à menor capacidade de enfrentamento das adversidades da doença.

É possível que a não detecção de associação significativa entre a resiliência e características demográficas no presente estudo deva-se ao número limitado de pacientes, uma vez que diverge de alguns estudos previamente publicados. Segundo Santos e Costa (2016), o relacionamento conjugal é considerado fundamental para o bem estar social e psicológico das pessoas, à medida que pode satisfazer as necessidades emocionais básicas do ser humano, fator que contribui para a 
superação das dificuldades na saúde e na qualidade de vida. Entretanto, esta associação não foi observada no presente estudo. Segundo Liu et al. (2018), avaliando a correlação da resiliência com o suporte social na depressão em pacientes em HD, o suporte social não foi um tampão significativamente efetivo sobre a gravidade dos sintomas depressivos, o que pode indicar a contribuição de fatores inerentes à personalidade do indivíduo no enfrentamento das dificuldades. Slomka (2011), ao avaliar a associação entre resiliência e o estadoclínico dos pacientes em diálise, observou a existência de uma associação positiva, porém de fraca intensidade, entre a resiliência e o IMC do paciente $(r=0,27, p=0,038)$. $\mathrm{O}$ autor considera que não há como afirmar se seria o elevado nível de resiliência de um paciente em HD o responsável por um comportamento de maior autocuidado do paciente em relação à sua dieta e ingesta alimentar, consequentemente, influenciando positivamente no IMC. Por outro lado, o nível educacional, econômico e a duração da doença renal foram fatores preditivos da resiliência de pacientes em diálise (Duran, Avci, Esim, 2020).

O tipo de estudo transversal, com um número pequeno de pacientes, de um único centro, que não objetivou avaliar o estado de resiliência de modo longitudinal e nem testar sua associação com complicações pós-diálise, como taxa de hospitalização e sobrevida do paciente são limitações do presente estudo. Estudos adicionais, prospectivos, com maior número de pacientes, e que investiguem correlação entre resiliência e morbimortalidade em diálise, estão indicados para esclarecer esta questão tão importante. A adoção de intervenções preventivas para o desenvolvimento de fatores promotores de resiliência poderá melhorar os resultados em saúde de pacientes hemodialíticos, devendo ser incentivada pela equipe multiprofissional.

\section{Conclusão}

Os pacientes em HD apresentaram nível moderado a elevado de resiliência em sua maioria, e quanto maior o nível de resiliência, menor o escore de depressão, ansiedade e de religiosidade intrínseca, o que reflete um estado afetivo mais positivo e uma maior frequência nas experiências espirituais e proximidade com Deus. Não houve associação com variáveis clínicas, laboratoriais ou sociodemográficas dos pacientes. A interação religiosidade, espiritualidade e alterações do estado de saúdemental com o estado de resiliência em hemodiálise precisa ser melhor pesquisada em estudos prospectivos e com maior número de pacientes, com o intuito de promover intervenções que possam auxiliar no manejo das dificuldades e adaptações à DRC.

\section{Agradecimentos}

Agradecemos à direção da Clínica de hemodiálise que autorizou a realização do estudo, bem como aos pacientes que aceitaram participar do estudo, cedendo seu tempo, disponibilidade e atenção para a coleta dos dados.

\section{Referências}

Angst, R. (2009). Psicologia e resiliência: Uma revisão de literatura. Psicol. Argum., 27(8): 253-260. 10.7213/rpa.v27i58.20225

Apóstolo, J. L. A., Mendes, A. C. \& Azeredo, Z. A. (2006). Adaptação para a língua portuguesa da depression, anxiety and stress scale (DASS). Rev. LatinoAm. Enfermagem [online], 14 (6): 863-871. http://dx.doi.org/10.1590/S0104-11692006000600006.

Berman, E., Merz, J. F., Rudnick, M., Rogers, K.K., Lee, J., et al. (2004). Religiosity in a hemodialysis population and its relationship to satisfaction with medical care, satisfaction with life, and adherence. Am J Kidney Dis., 44(3): 488-97. doi:https://doi.org/10.1053/j.ajkd.2004.05.027.

Boell, J. E. W., da Silva, D. M. G. V. \& Hegadoren, K. M. (2016). Sociodemographic factors and health conditions associated with the resilience of people with chronic diseases: a cross sectional study. Rev. Latino-Am. Enfermagem, 24: e2786. https://doi.org/10.1590/1518-8345.1205.2786.

Bujang, M. A., Musa, R., Liu, W. J., Chew, T. F., Lim, C. T. S, \& Morad, Z. (2015). Depression, anxiety and stress among patients with dialysis and the association with quality of life. Asian J Psychiatr, 18:49-52. 10.1016/j.ajp.2015.10.004. 
Burlacu, A., Artene, B., Nistor, I., Buju, S., Jugrin, D., Mavrichi, I. \& Covic, A. (2019). Religiosity, spirituality and quality of life of dialysis patients: a systematic review. Int Urol Nephrol., 51(5):839-850. 10.1007/s11255-019-02129-x.

Cal, S. F., de Sá, L. R., Glustak, M. E. \& Santiago, M. B. (2015) Resilience in chronic diseases: A systematic review. Cogent Psychology, 2:1. 10.1080/23311908.2015.1024928

Carone Jr, D. A. \& Barone, D. F. (2001). A social cognitive perspective on religious beliefs: their functions and impact on coping and psychotherapy. Clin Psychol. Rev., 21(7): 989-1003. 10.1016/s0272-7358(00)00078-7.

Censo Brasileiro de 2010. Rio de Janeiro: IBGE, 2012. Instituto Brasileiro de Geografia e Estatística (IBGE).

Connor, K. M. \& Davidson, J. R. T. (2003). Development of a new Resilience Scale: The Connor-Davidson Resilience Scale (Cd-Risc). Depress. Anxiety, 18(2):76-82. 10.1002/da.10113.

Correia, A. L. R., Barbosa, I. V, Lima, F. E. T., et al. (2015). Utilização da Escala de avaliação da espiritualidade em pacientes portadores de lesão renal em hemodiálise. Cogitare Enferm., 20 (3): 489-95.: http://dx.doi.org/10.5380/ce.v20i3.40816

Damásio, B. F., Borsa, J. C. \& Silva, J. P. 14-Item Resilience Scale (RS-14): Psychometric Properties of the Brazilian Version. (2011). J Nurs Meas., 19 (3): 131-45. 10.1891/1061-3749.19.3.131.

D’Souza, R. F. \& Rodrigo, A. doi (2004). Spiritually augmented cognitive behavioural therapy. Australas Psychiatry, 12 (2):148-152. 10.1080/j.10398562.2004.02095.x

Duran, S., Avci, D. \& Esim, F. (2020). Association Between Spiritual Well-Being and Resilience Among Turkish Hemodialysis Patients. J Relig Health., 59(6):3097-3109. 10.1007/s10943-020-01000-z.

Fassbinder, T. R. C., Winkelmann, E. R., Schneider, J., Wendland, J. \& Oliveira, O. B. (2015). Capacidade funcional e qualidade de vida de pacientes com doença renal crônica pré-dialítica e em hemodiálise - Um estudo transversal. J Bras Nefrol., 37 (1): 47-54. https://doi.org/10.5935/0101-2800.20150008

Freire de Medeiros, C. M., Arantes, E. P., Tajra, R. D., Santiago, H. R., Carvalho, A. F. \& Libório, A. B. (2017) Resilience, religiosity and treatment adherence in hemodialysis patients: a prospective study. Psychol Health Med., 22(5): 570-577. 10.1080/13548506.2016.1191658.

Forti, S., Serbena, C. A. \& Scaduto, A. A. (2020). Mensuração da espiritualidade/religiosidade em saúde no Brasil: uma revisão sistemática. Ciênc. Saúde Coletiva, 25(4): 1463-1474. https://doi.org/10.1590/1413-81232020254.21672018.

Gomes, C. C. I., Lanzotti, B. R. \& Orlandi, S. F. (2017). Resilience in Patients with Chronic Kidney Disease in Hemodialysis. International Journal of Nursing and Health Sciences, 11(5). https://zenodo.org/badge/DOI/10.5281/zenodo.1130415.svg

Kimura, M., Oliveira, A., Mishima, L. \& Underwood, L. (2012). Adaptação cultural e validação da Underwood's Daily Spiritual Experience Scale - versão brasileira. Rev Esc Enferm USP [online], 46 (n. esp.):99-106. https://doi.org/10.1590/S0080-62342012000700015

Levin, J. \& Fox, J.A. (2004). Estatística para ciências humanas. São Paulo: Prentice Hall.

Liu, Y. M., Chang, H. J., Wang, R. H., Yang, L. K., Lu, K. C. \& Hou, Y. C. (2018). Role of resilience and social support in alleviating depression in patients receiving maintenance hemodialysis. Ther Clin Risk Manag., 14, 441-451. 10.2147/TCRM.S152273.

Lovibond, S. H. \& Lovibond, P. F. (1995). Manual for the depression anxiety stress scales. Psychology Foundation.

Lucchetti, G., Almeida, L. G. C. \& Granero, A. L. (2010). Espiritualidade no paciente em diálise: o nefrologista deve abordar? J Bras Nefrol., 32(1):128-32. https://doi.org/10.1590/S0101-28002010000100020

Marinho, A. W. G. B., Penha, A. P., Silva, M. T. \& Galvão, T. F. (2017). Prevalência de doença renal crônica em adultos no Brasil: revisão sistemática da literatura. Cad. saúde colet. [online]., 25(3):379-388. https://doi.org/10.1590/1414-462x201700030134

Martinez, E. Z., Alves, A. C., Carneiro, A. F. T. M. et al. (2014). Investigação das propriedades psicométricas do Duke Religious Index no âmbito da pesquisa em saúde coletiva. Cad. Saúde Colet. [online], 22 (4): 419-27. https://doi.org/10.1590/1414-462X201400040016.

Murtagh, F. E., Addington-Hall, J. \& Higginson, I. J. (2007). The prevalence of symptoms in end-stage renal disease: a systematic review. Adv. Chronic Kidney Dis., 14 (1): 82-99. 10.1053/j.ackd.2006.10.001.

Oliveira, C. M., Costa, S. P., Costa, L. C., Pinheiro, S. M., Lacerda, G. A. \& Kubrusly, M. (2012). Depression in dialysis patients and its association with nutritional markers and quality of life. J Nephrol., 25(6):954-61. 10.5301/jn.5000075.

Ottaviani, A. C., Souza, E. N., Drago, N. C., Mendiondo, M. S. Z., et al. (2014). Esperança e espiritualidade de pacientes renaiscrônicos em hemodiálise: estudo correlacional. Rev. Latino-Am. Enfermagem [on-line], 22 (2): 248-254. https://doi.org/10.1590/0104-1169.3323.2409

Pesce, R. P., Assis, S. G., Avanci, J. Q., Santos, C. N., Malaquias, J. V. \& Carvalhaes, R. (2005). Adaptação transcultural, confiabilidade e validade da escala de resiliência. Cadernos de Saúde Pública, 21(2), 436-448. https://doi.org/10.1590/S0102-311X2005000200010.

Santos, P. R., Capote Júnior, J. R. F. G, Cavalcante Filho, J. R. M., Ferreira, T. P., Santos Filho, J. N. G. \& Oliveira, S. S.(2017). Religious coping methods predict depression and quality of life among end-stage renal disease patients undergoing hemodialysis: a cross-sectional study. BMC Nephrol., 18(1):197. $10.1186 / \mathrm{s} 12882-017-0619-1$

Santos, R. I. \& Costa, O. R. S. (2016). Avaliação da resiliência em pacientes com Insuficiência Renal Crônica submetidos à hemodiálise. Rev. Cienc. Saúde, 6 (1): 5-13. https://doi.org/10.21876/rcsfmit.v6i1.461 
Research, Society and Development, v. 10, n. 7, e27110716106, 2021

(CC BY 4.0) | ISSN 2525-3409 | DOI: http://dx.doi.org/10.33448/rsd-v10i7.16106

Siqueira, J., Fernandes, N. M. \& Moreira-Almeida, A. (2019). Associação entre religiosidade e felicidade em pacientes com doença renal crônica em hemodiálise. J Bras Nefrol., 41(1): 22-28. https://doi.org/10.1590/2175-8239-jbn-2018-0096

Slomka, L.(2011). Associação entre o nível de resiliência e o estado clínico de pacientes renais crônicos em hemodiálise. Barbarói, (34): 23-37. http://pepsic.bvsalud.org/scielo.php?script=sci_arttext\&pid=S0104-65782011000100003\&lng=pt\&tlng=pt.

Souza, J. G., Devezas, A. M. L. O. \& Santos, L. C. S. (2018). Avaliação da espiritualidade e religiosidade de pacientes com doença renal crônica em hemodiálise. Arq Med Hosp Fac Cienc Med Santa Casa São Paulo, 63(3):160-7. https://doi.org/10.26432/1809-3019.2018.63.3.160.

Taunay, T. C. D. E, Gondim, F. A. A., Macêdo, D. S., Moreira-Almeida, A., et al. (2012). Validação da versão brasileira da escala de religiosidade de Duke (DUREL). Rev Psiquiatr Clín., 39 (4): 130-5. http://dx.doi.org/10.1590/S0101-60832012000400003

Underwood, L. G. \& Teresi, J. (2002). The Daily Spiritual Experience Scale: development, theoretical, description, reliability, exploratory factor analysis and preliminary construct validity using health related data. Ann Behav Med., 24(1):22-33. 10.1207/S15324796ABM2401_04.

Wagnild, G. M. \& Young, H. (1993). Development and psychometric evaluation of the Resilience Scale. J Nurs Meas., 1 (2):165-178.

Wagnild G. M. (2009) A review of the Resilience Scale. J Nurs Meas., 17(2):105-13. 10.1891/1061-3749.17.2.105.

Wagnild, G. M. (2009a). The Resilience Scale user's guide for the US English version of The Resilience Scale and The 14-Item Resilience Scale (RS-14). Worden, MT: The ResilienceCenter.

Zimmermann, P. R., Carvalho, J. O. \& Mari, J. J. (2004). Impacto da depressão e outros fatores psicossociais no prognóstico de pacientes renais crônicos. Rev. Psiquiatr. Rio Gd Sul, 26 (3): 312-318. https://doi.org/10.1590/S0101-81082004000300008. 